\section{Práticas pessoais e profissionais de promoção da atividade física em agentes comunitários de saúde}

\author{
Personal and professional practices of \\ physical activity promotion in community \\ health workers
}

Taynã Ishii dos Santos ${ }^{1,3}$

Paulo Henrique Guerra ${ }^{2,3}$

Douglas Roque Andrade ${ }^{2,3}$

Alex Antonio Florindo 1,2,3

\section{RESUMO}

Os Agentes Comunitários de Saúde (ACS) são profissionais importantes na promoção da atividade física (AF) no Sistema Único de Saúde. No entanto, poucos estudos têm investigado o nível de AF e práticas profissionais de ACS na promoção da AF. Por este motivo, a pesquisa objetiva verificar a relação entre práticas profissionais de promoção da $\mathrm{AF}$ com o nível de AF em um grupo de ACS trabalhadores de uma unidade de saúde da zona leste da cidade de São Paulo. Estudo realizado com 30 ACS sobre nível de AF (questionário e pedômetro) e as práticas profissionais para a promoção da $\mathrm{AF}$ sobre recomendação e participação em grupos de AF oferecidos para usuários da UBS. Foram realizadas análises descritivas e análises de diferenças de médias por meio do teste $\mathrm{t}$ de Student para amostras independentes no software SPSS (versão 15.0). Como resultado a maioria das ACS não praticava pelo menos 150 minutos de $\mathrm{AF}$ no lazer e não atingiu a recomendação de pelo menos 10 mil passos diários de caminhada. Quanto à prática profissional 50,0\% relataram recomendar AF para os usuários há pelo menos seis meses e 53,6\% relataram liderar ou participar de grupos de AF para usuários. As ACS que recomendavam AF para os usuários tiveram maior média de passos diários de caminhada $(\mathrm{p}=0,039)$ em comparação com as que não recomendavam. A prática profissional de aconselhamento teve associação com a prática de AF neste grupo, demonstrando a importância de incentivar a promoção da $\mathrm{AF}$ para os trabalhadores da área da saúde.

\section{PALAVRAS-CHAVE}

Agentes Comunitários de Saúde; Estilo de vida; Prática profissional; Atividade física.

\begin{abstract}
The Community Health Workers (CHWs) are important professionals to promote physical activity (PA) on within the Brazilian Unified Health Service. However, few studies have investigated lifestyle-related health indicators and professional practices associated with PA promotion among $C H W$ s. For this reason, this research has evaluated the association between professional practices related with $P A$ promotion with $P A$ level in a group of $C H W$ s who were working in a primary bealthcare unit (PHU) of the city of São Paulo. This study was realized with 30 CHWs about level of PA (questionnaire and pedometer) and professional practices for the promotion of PA (on recommendation and participation in PA groups offered to PHU users). Descriptive and mean comparison analyses were performed using the Student's t test for independent in SPSS software (version 15.0). The results showed that $C H W$ s did not practice at least 150 minutes of $P A$ in leisure and did not reach the recommendation of at least 10 thousand steps daily walk. As for the professional practice $51.9 \%$ reported recommend $P A$ to users for at least six months and $53.6 \%$ reported leading or participating $P A$ groups for users. The CHWs that recommend $P A$ for users had higher mean daily steps walk ( $P$ $=0.039$ ) compared with those not recommended. The professional counseling practice was associated with the practice of PA in this group, demonstrating the importance of encouraging the promotion of $P A$ for workers in the bealth area.
\end{abstract}

\section{KEYWORDS}

Community Health Workers; Lifestyle; Professional Practice; Physical Activity.
Rev Bras Ativ Fís Saúde p. 165-173 DOI

http://dx.doi.org/10.12820/rbafs.v.20n2p165

1 Faculdade de Saúde Pública - Universidade de São Paulo, Departamento de Nutrição, São Paulo/ $S P$ - Brasil.

2 Escola de Artes, Ciências e Humanidades Universidade de São Paulo, São Paulo/SP - Brasil. 3 Grupo de Estudos e Pesquisas Epidemiológicas em Atividade Física e Saúde, Escola de Artes, Ciências e Humanidades da Universidade de São Paulo (GEPAF/EACHUSP). 


\section{INTRODUCCÃO}

Buscando reverter o quadro da baixa prevalência da prática de atividade física (AF) em adultos brasileiros tanto no tempo de lazer, como na forma de deslocamento ${ }^{1}$, o Sistema Único de Saúde (SUS) intensificou o desenvolvimento e a disseminação de ações, programas e políticas para promover a $\mathrm{AF}$ no nível populacional. Considerando que, aproximadamente $80 \%$ da população brasileira depende exclusivamente do atendimento oferecido pelo SUS e a inserção da prática de AF enquanto um dos fatores determinantes da saúde no trabalho de promoção da saúde e prevenção de doenças ${ }^{2,3}$, este cenário amplia a perspectiva de um impacto benéfico gradual nos próximos anos para a saúde pública.

Uma das formas utilizadas para promover AF no SUS é por meio de aconselhamentos, que podem ser caracterizados como uma breve intervenção por meio de conversa básica feita por profissionais da saúde com os usuários e objetiva alterar algum comportamento negativo para a saúde ou reforçar comportamentos positivos ${ }^{4}$. No caso da promoção da AF, o aconselhamento é reconhecido como uma estratégia de ação de saúde e educação para desenvolver autonomia, enfrentamento de problemas e adoção de mudanças de comportamento que sejam condizentes com a promoção da saúde ${ }^{5}$. Neste sentido, alguns estudos apresentam a efetividade do aconselhamento na atenção primária, tanto na promoção da alimentação saudável ${ }^{6}$ quanto na promoção da $\mathrm{AF}{ }^{7,8}$.

Um trabalho que sugere que o aconselhamento deve ser uma prática de rotina nos sistemas de saúde foi o estudo realizado por Florindo et al. ${ }^{9} \mathrm{com}$ amostra representativa de médicos e enfermeiros que atuavam na atenção básica à saúde do Brasil, demonstrando que 69\% destes profissionais aconselhavam prática de AF para usuários do sistema há mais de seis meses, provando ser uma prática recorrente entre os profissionais do SUS. Este estudo também mostrou que os fatores associados ao aconselhamento foram: avaliação do nível de AF dos usuários; não ter falta de tempo para aconselhar como barreira; se sentir preparado para aconselhar; e UBS que ofereciam programas de $\mathrm{AF}$ para os usuários. No entanto, os autores não estudaram a relação do hábito de praticar AF com a conduta profissional de aconselhar este comportamento.

Por outro lado, evidências recentes apontam que os volumes de atividade física apresentados pelos profissionais da saúde podem influir em sua prática de aconselhamento de atividade física. Ao mesmo tempo profissionais que atingem a recomendação de prática de $\mathrm{AF}$ do American College of Medicine and Sports aconselham mais AF para seus pacientes e usuários do sistema de saúde ${ }^{10}$, onde profissionais com baixos níveis de AF orientam menos para a prática aos usuários ${ }^{11}$. Nesse sentido, uma das possíveis saídas para reverter este quadro seria estimular a mudança de comportamento destes profissionais, acarretando em benefícios tanto para eles mesmos, quanto para as pessoas que se submetem ao seu atendimento.

Entretanto, existem poucos estudos verificando os níveis de AF e suas relações com as práticas profissionais como aconselhamentos ou participação em grupos de AF em Agentes Comunitários de Saúde (ACS), que são profissionais muito importantes na Estratégia de Saúde da Família (ESF), principalmente 
por serem os mediadores entre a comunidade e as equipes de saúde, moradores da comunidade em que atuam, compartilharem das mesmas situações dos usuários do SUS no território e por serem referência para diversas ações domiciliares e comunitárias ${ }^{12,13}$. Na revisão de literatura desde artigo, encontrou-se apenas um estudo com ACS da região de São Caetano do Sul, o qual que investigou o nível de atividade física por pedômetros e concluiu que todos os ACS das equipes que participavam como profissionais auxiliando num programa de promoção da atividade física atingiram a média diária de $10 \mathrm{mil}$ passos ${ }^{14}$.

Nesse sentido, justificado pela possível existência de associação proporcional entre hábitos de vida e atitudes profissionais e do pequeno número de trabalhos que abordam essa relação nos ACS, o objetivo deste estudo foi verificar se existe relação entre práticas profissionais de promoção da $\mathrm{AF}$ com o nível de AF em um grupo de ACS trabalhadoras de uma unidade de saúde da zona leste da cidade de São Paulo.

\section{MÉTODOS}

Este estudo foi do tipo transversal e fez parte de uma pesquisa maior denominada de "Ambiente Ativo" que teve como objetivo avaliar intervenções de promoção da atividade física no SUS por meio da ESF, partindo do modelo lógico proposto por Andrade et al. ${ }^{15}$. Fizeram parte deste estudo 30 ACS do sexo feminino que trabalhavam em uma UBS com ESF no distrito de Ermelino Matarazzo, zona leste de São Paulo, no ano de 2011.

Foram avaliadas as seguintes variáveis sociais, demográficas e de hábitos de vida: idade, cor da pele, estado marital, escolaridade, tempo de trabalho na UBS, tabagismo, percepção de saúde e qualidade de vida.

A prática de atividades físicas moderadas e vigorosas no tempo de lazer foi avaliada por meio do questionário internacional de atividade física ou International Physical Activity Questionnaire (IPAQ) versão longa, de acordo com as recomendações de Hallal et al. ${ }^{16}$, este instrumento foi aplicado em forma de entrevista. $\mathrm{O}$ parâmetro escolhido para determinar o nível mínimo de atividade física com fins na promoção da saúde foi a recomendação de 150 minutos semanais de $\mathrm{AF}$ moderadas e vigorosas.

A caminhada foi avaliada de forma objetiva por meio de pedômetros $\mathrm{Di}$ giwalker modelo CW 700. As ACS receberam instruções para utilização do pedômetro durante sete dias consecutivos e foram orientadas para retirá-los somente durante o banho, nas atividades aquáticas e para dormir, de acordo com as recomendações de Tudor-Locke e Bassett $\mathrm{Jr}^{17}$.

As práticas profissionais de promoção da atividade física foram avaliadas por meio de duas questões: 1) Se as ACS recomendavam atividade física para os usuários há pelo menos seis meses (sim; não); 2) Se as ACS participavam ou colaboravam com grupos de promoção da atividade física realizados na UBS onde trabalhavam (sim; não) ${ }^{18}$.

Foram realizadas análises de frequências das características sociais, demográficas, de hábitos e das práticas profissionais de promoção da atividade física considerada neste estudo como variável dependente. E análises descritivas de médias e desvios-padrão (dp) e dos valores mínimos e máximos dos minutos por semana de prática de atividade física no tempo de lazer e de passos caminhados. 
Para verificar distribuição de aderência à curva normal das variáveis quantitativas utilizou-se o teste Kolmogorov-Smirnov.

Posteriormente, procedeu-se o cálculo das diferenças de médias dos minutos de prática de atividade física no tempo de lazer e da média diária do número de passos caminhados segundo práticas profissionais de promoção da atividade física (recomendação regular: sim ou não; participação ou colaboração em grupos de promoção da atividade física nas UBS: sim ou não), utilizando-se o teste t de Student para amostras independentes ou seu equivalente não paramétrico para amostras independentes (teste de Mann-Whitney).

Todas as variáveis foram duplamente digitadas e conferidas no módulo validate do programa EpiData 3.1 e todas as análises foram realizadas no programa Statistical Package for the Social Sciences (SPSS), versão 15.0.

A pesquisa foi aprovada pelo Comitê de Ética da Secretaria Municipal de Saúde de São Paulo sob o protocolo CAEE 0072.0.162.000-10 e este estudo foi aprovado pelo Comitê de Ética da Faculdade de Saúde Pública da Universidade de São Paulo sob o número do processo OF.COEP/047/13.

\section{RESULTADOS}

Fizeram parte deste estudo 30 ACS do sexo feminino que trabalhavam em uma UBS com ESF no distrito de Ermelino Matarazzo, zona leste de São Paulo, no ano de 2011. A maioria das entrevistadas tinha a idade entre 18 a 39 anos, escolaridade até o ensino médio completo, cor da pele preta ou parda, eram casadas, não fumavam, a maioria declarou ter uma saúde boa ou muito boa e trabalhava há menos de cinco anos na unidade (Tabela 1).

Sobre os níveis de atividade física (Tabela 2), a média semanal de prática de atividade física no lazer foi de pouco mais de uma hora, mas com variação de nenhuma prática a até mais de sete horas por semana. Ressalta-se que apenas $18,5 \%$ das ACS atingiram pelo menos 150 minutos por semana de prática no lazer (dados não mostrados).

A média diária de passos também teve grande variação (Tabela 2), com ACS com média muito baixa de menos de 200 passos até superiores a $10 \mathrm{mil}$ passos diários. Ressalta-se que 33,3\% das ACS atingiram a média diária dos 10.000 passos por dia de caminhada (dados não mostrados).

Metade das ACS recomendava atividade física para os usuários e colaborava ou participava de grupos de promoção da atividade física desenvolvidos nas UBS (Figura 1).

Com relação às diferenças de médias de prática de atividade física segundo a realização de práticas profissionais de promoção (Tabela 3), as ACS que recomendam atividade física há mais de seis meses para os usuários do SUS tiveram maior média de passos diários de caminhada. 
TABELA 1 - Distribuição por frequência das ACS segundo as características sociodemográficas, profissionais e variáveis do estilo de vida. Ermelino Matarazzo, SP, 2011.

\begin{tabular}{|c|c|c|}
\hline Variáveis & & $\%$ \\
\hline \multicolumn{3}{|l|}{ Idade (anos) } \\
\hline & 18 a 39 & 55,6 \\
\hline & 40 anos ou mais & 44,4 \\
\hline \multicolumn{3}{|l|}{ Cor da pele } \\
\hline & Brancas & 40,7 \\
\hline & Pretas ou Pardas & 59,3 \\
\hline \multicolumn{3}{|l|}{ Escolaridade } \\
\hline & Do ensino fundamental até o ensino médio completo & 88,9 \\
\hline & Ensino superior incompleto ou mais & 11,1 \\
\hline \multicolumn{3}{|l|}{ Estado Marital } \\
\hline & Casada ou com companheirolal & 64,0 \\
\hline & Solteira ou sem companheirolal & 36,0 \\
\hline \multicolumn{3}{|c|}{ Tempo de trabalho na UBS } \\
\hline & Menos de cinco anos & 67,9 \\
\hline & Cinco anos ou mais & 32,1 \\
\hline \multicolumn{3}{|l|}{ Tabagismo } \\
\hline & Não fuma & 80,0 \\
\hline & Fuma & 20,0 \\
\hline \multicolumn{3}{|l|}{ Autorrelato de saúde } \\
\hline & Regular, ruim ou muito ruim & 36,0 \\
\hline & Boa ou muito boa & 64,0 \\
\hline
\end{tabular}

TABELA 2 - Distribuição por médias, desvios-padrão e valores mínimos e máximos, da prática de atividade física no tempo de lazer e do número de passos diários caminhados de ACS trabalhadoras de uma UBS de Ermelino Matarazzo, SP, 2011.

\begin{tabular}{lccc}
\hline Variáveis & Média (dp) & $\begin{array}{c}\text { Valores } \\
\text { mínimos }\end{array}$ & $\begin{array}{c}\text { Valores } \\
\text { máximos }\end{array}$ \\
\hline Atividade física no tempo de lazer (minutos/semana) & $76,5(101,4)$ & -- & 435,0 \\
\hline Passos (números/dia) & $7946,0(3380,7)$ & 142,0 & 13852,4 \\
\hline
\end{tabular}

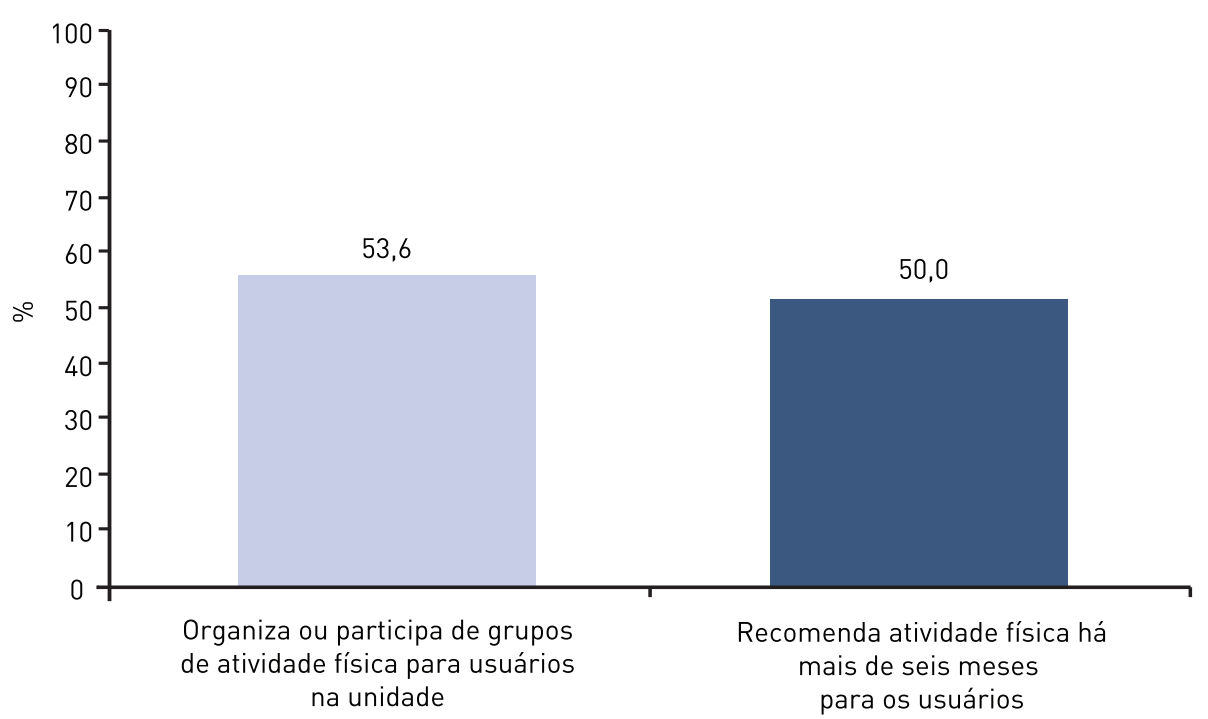

FIGURA 1 - Práticas profissionais relacionadas à promoção da atividade física de Agentes Comunitárias de Saúde. Ermelino Matarazzo, SP, 2011. 
TABELA 3 - Distribuição contínua por médias e desvios-padrão das variáveis de atividade física segundo prática profissional de promoção de AF atividade física de um grupo de ACS trabalhadoras de uma UBS de Ermelino Matarazzo, SP, 2011.

\begin{tabular}{lccc}
\hline Variáveis & média (dp) & \multicolumn{1}{c}{ média (dp) } & \\
\hline $\begin{array}{l}\text { Recomendação de AF para usuários há mais de } \\
\text { seis meses }\end{array}$ & Não & Sim & \\
\hline AF no tempo de lazer (minutos/semana) & $75,0(125,3)$ & $89,6(82,1)$ & 0,731 \\
\hline $\begin{array}{l}\text { Média diária de passos caminhados } \\
\text { Organização ou participação em grupos de } \\
\text { promoção da AF para usuários }\end{array}$ & $6417,4(3239,8)$ & $9265,2(3263,6)$ & $0,039 *$ \\
\hline AF no tempo de lazer (minutos/semana) & $81,8(129,0)$ & Sim & \\
\hline Média diária de passos caminhados & $6514,2(3400,1)$ & $8985,7(32,4)$ & 0,974 \\
\hline
\end{tabular}

\section{DISCUSSÃO}

Os principais resultados deste estudo mostraram que a maioria das ACS avaliadas não praticavam 150 minutos de $\mathrm{AF}$ moderadas e vigorosas no lazer e nem dos 10 mil passos por dia de caminhada. Metade das ACS entrevistadas aconselhavam usuários a praticarem $\mathrm{AF}$ e participavam de grupos de $\mathrm{AF}$ na unidade de saúde. O grupo que aconselhava os usuários a praticarem $\mathrm{AF}$ teve maior média de passos caminhados diariamente.

Os achados apresentados no presente artigo divergem dos apresentados pelo estudo de Silva et al. ${ }^{14}$, em que todos os ACS de uma unidade básica de saúde de São Caetano do Sul atingiam a recomendação de pelo menos $10 \mathrm{mil}$ passos por dia. É importante ressaltar que estes ACS participaram como profissionais de um programa que teve como objetivo promover AF praticando junto com a população.

Estes resultados são preocupantes, pois a prática de atividade física regular é importante para melhora da saúde global de um indivíduo, uma vez que reduz riscos de doenças cardiovasculares e mortalidade precoce ${ }^{19}$. Segundo estudos laborais com diferentes amostras de grupos de ACS no Brasil, foi mostrado que a qualidade de vida destes profissionais é afetada negativamente pelo estresse causado pelas características de seu trabalho ${ }^{20,21}$. Neste sentido, a prática de $\mathrm{AF}$ no lazer poderia auxiliar no autocuidado, que pode tornar-se um modo para amenizar as cargas impostas pela alta demanda e tarefas diárias e por ser uma atividade de promoção da saúde que pode ser estendida para o cuidado com os usuários.

Existem poucos estudos na literatura que investigaram a relação entre níveis de $\mathrm{AF}$ e a prática de aconselhamento, porém, especialistas como Oberg e Frank ${ }^{22}$ descrevem que é muito importante o aconselhamento realizado por profissionais de saúde na atenção primária que possuem comportamentos saudáveis, pois estes profissionais são reconhecidos como fontes seguras de informações e como modelos de saúde.

Esta pesquisa mostrou que o grupo de ACS que aconselhava AF para as pessoas teve uma média maior em número de passos caminhados diariamente em comparação com o grupo que não praticava. Outros estudos têm encontrado resultados similares. Por exemplo, Frank et al. demonstraram que há uma relação entre o hábito de se praticar AF e o aconselhamento para se promover este comportamento ${ }^{10}$. Os autores realizaram uma pesquisa nacional nos Estados Unidos com mais de 4.500 médicas e constataram que a prática de exer- 
cícios físicos foi associada positivamente com a frequência de aconselhamento sobre os benefícios da AF para pacientes. Em enfermeiras do Reino Unido ocorreu o mesmo fenômeno, sendo que aquelas que se exercitavam regularmente eram mais propensas a incentivar a $\mathrm{AF}$, relataram menos fatores limitantes a promover $\mathrm{AF}$, aconselhavam mais frequentemente e para diferentes grupos de pacientes ${ }^{23}$.

Em uma intervenção educacional de quatro anos com estudantes de medicina estadunidenses realizado por Frank et al. ${ }^{24}$, resultou em um maior aconselhamento da prática de AF em comparação com o grupo controle, porém, não houve diferença significativa entre os grupos com relação à prática de $\mathrm{AF}$, demonstrando que aprender sobre $\mathrm{AF}$ aumenta e melhora o aconselhamento, mas não é o suficiente para alterar o comportamento dos estudantes.

Com relação aos ACS, o único estudo encontrado na revisão de literatura mostrou que o nível de AF destes profissionais que participaram de uma intervenção $\mathrm{AF}$ aconselhando os usuários a aderirem a programas foi alto. Neste estudo os ACS também tiveram um espaço de convivência para que pudessem praticar, os mesmos passaram por um treinamento sobre promoção da $\mathrm{AF}$ e também realizavam AF orientadas por um profissional de Educação Física, assim como os usuários que eles eram os responsáveis por convidar as pessoas a participarem dessas aulas. $\mathrm{O}$ estudo mostrou que o número médio de passos por dia destes profissionais foi maior em comparação com a amostra deste estudo e todos atingiram a recomendação de 10 mil passos por dia ${ }^{14}$.

A principal limitação deste estudo foi o pequeno número de ACS que foram estudadas. Este tamanho de amostra prejudicou algumas análises de diferenças de médias. Um cálculo de tamanho de amostra feito a posteriori no programa GPOWER 3.1.9.2 com os resultados significativos das diferenças obtidas entre o número médio diário de passos e os dp dos grupos de ACS que participavam e que não participavam de grupos de atividade física nas UBS, mostrou um tamanho de efeito de Cohen $=0,74$. Supondo-se um alfa $=5 \%$ e um beta $=80 \%$ para um teste bicaudal, com este tamanho de efeito seriam necessários pelo menos $60 \mathrm{ACS}$, ou seja, o dobro da amostra analisada. Isso prejudicou as análises de diferenças de médias. No entanto, mesmo com este tamanho de amostra reduzido, conseguiu-se mostrar uma diferença estatística importante indicando que as ACS que fazem mais AF, também aconselham mais AF, o que poderia ser maximizado se a amostra fosse maior.

Além disso, estudos transversais não estabelecem temporalidade entre as variáveis. Nesse sentido, tornam-se limitadas inferências de causalidade entre as práticas profissionais para a promoção da atividade física e a realização da caminhada realizada pelas ACS. No entanto, a presente pesquisa, desde seu delineamento, limitou-se ao levantamento dos níveis de AF e práticas de aconselhamento oferecidas pelas ACS.

No entanto, apesar das limitações, recomenda-se que sejam feitas intervenções para a promoção da atividade física com ACS para que, além de beneficiar a saúde destes profissionais, acredita-se que este tipo de ação possa resultar em benefício para o aconselhamento de promoção da AF para usuários do SUS.

Como conclusão, os resultados obtidos neste grupo de ACS que trabalhava numa unidade de saúde da zona leste de São Paulo mostrou que a maioria das profissionais não atingia a recomendação de $\mathrm{AF}$ moderadas e vigorosas no lazer $(81,5 \%)$ ou do número de passos por dia em caminhada $(66,7 \%)$. 
Observou-se que metade das profissionais aconselhavam os usuários do SUS a praticarem AF e também participava de grupos de AF na unidade. As ACS que aconselhavam os usuários a praticarem AF apresentaram maior média de número de passos por dia se comparadas às que não realizavam aconselhamento.

\section{Contribuição dos autores}

TIS participou na coleta dos dados e elaboração do texto. PHG e DRA contribuíram com a escrita e revisão do texto. AAF idealizou o projeto principal e participou de todas as etapas desse trabalho.

\section{Financiamento}

Estudo financiado pela FAPESP (auxílio à pesquisa 2009/14119-4). A primeira autora deste artigo foi bolsista CAPES (Processo 00.889.834/0001-08).

\section{REFERÊNCIAS}

1. Florindo AA, Guimarães VV, Cesar CL, Barros MB, Alves MC, Goldbaum M. Epidemiology of leisure, transportation, occupational and household physical activity: prevalence and associated factors. J Phys Act Health. 2009; 6(5) 625-32.

2. Paim J, Travassos C, Almeida C, Bahia L, Macinko. Saúde no Brasil 1 O sistema de saúde brasileiro: história, avanços e desafios. The Lancet. 2012; 377 (9779): 11-31.

3. Brasil, Ministério da Saúde. Programa saúde da família: ampliando a cobertura para consolidar a mudança do modelo de atenção básica . Rev Bras Saúde Mater Infant. 2003; 3(1): p. 1113-25.

4. National Institute for Health and Clinical Excellence. Four commonly used methods to increase physical activity. http://guidance.nice.org.uk/ph2; March 2006.

5. Conselho Regional de Educação Física [internet]. São Paulo; 2014. [acesso em 22 de dezembro de 2014].Disponível em http://www. crefsp.org.br/interna.asp? campo=2157\&secao_id $=112$

6. Spencer EH, Frank EE, Lisa KH, Vicki SS, Mary KG, Deborah A. Predictors of nutrition counseling behaviors and attitudes in US medical students. Am J Clin Nutr. 2006; 84(3): 655-62.

7. Aittasalo M, Miilunpalo S, Kukkonen-Harjula K, Pasanen M. A randomized intervention of physical activity promotion and patient self-monitoring in primary health care. Prev Med. 2006; 42(1): 40-6.

8. Group for the Activity Counseling Trial Research Group. Effects of physical activity counseling in primary care: The Activity Counseling Trial: A randomized controlled trial. JAMA. 2001, 286 (6):677-687

9. Florindo AA, Costa EF, Sa TH, Santos TI, Velardi M, Andrade DR, Physical Activity Promotion in Primary Health Care in Brazil: A Counseling Model Applied to Community Health Workers. J Phys Act Health. 2014. No prelo.

10. Frank E, Wright EH, Serdula MK,Elon LK,Baldwin G. Personal and professional nutrition-related practices of US female physicians. Am J Clin Nutr. 2002; 75(2): 326-32.

11. Siqueira FCV, Nahas MC, Facchini LA, Piccini RX, Tomasi E, Thumé E, et al. Atividade física em profissionais de saúde do Sul e Nordeste do Brasil Physical activity among health professionals from South and Northeast Brazil. Cad Saúde Publ. 2009; 25(9): 1917-28.

12. Brasil, Ministério da Saúde. Portaria n 1.886 , de 18 de dezembro de 1997. Aprova as normas e diretrizes do programa de Agentes Comunitários de Saúde e do Programa de Saúde da Família. Brasília (DF); 1997.

13. Brasil, Ministério da Saúde. Cartilha entendendo o SUS. Brasília (DF); 2007.

14. Silva L, Matsudo S, Lopes G. Do diagnóstico à ação: Programa comunitário de atividade física na atenção básica: a experiência do município de São Caetano do Sul, Brasil. Rev Bras Ativ Fís Saúde. 2012; 16(1): 84-8. 
15. Andrade DR, Costa EF, Ribeiro EHC, Salvador EP, Garcia LMT, Florindo AA. Do diagnóstico à ação: A experiência da pesquisa Ambiente Ativo na promoção da atividade física em Ermelino Matarazzo, na zona leste de São Paulo, SP. Rev Bras Ativ Fís e Saúde. 2012; 17(3): 235-8.

16. Hallal PC, Gomez LF, Parra DC, Lobelo F, Mosquera J, Florindo AA, et al. Lições Aprendidas Depois de 10 Anos de Uso do IPAQ no Brasil e Colômbia. J Phys Act Health. 2010; 7(Suppl 2): S259-64.

17. Tudor-Locke C, Bassett Jr DR. How many steps/day are enough? Sports Med. 2004; 34(1): 1-8.

18. Sá,TH. Construção e avaliação de um programa educativo para a promoção de atividade física junto a equipes de saúde da família [dissertação]. 2011, São Paulo: Universidade de São Paulo, 2011.

19. Coleman KJ, Farrell MA, Rocha DA, Hayashi T,Hernandez M,Wolf J, et al. Readiness to be physically active and self-reported physical activity in low-income Latinas, California WISEWOMAN, 2006-2007. Prev Chronic Dis. 2012; 9: E87.

20. Dissen CM, Becke CLC, Prestes FC, Tavares JP, Vogi MS, Coelho APF. Cargas físicas no trabalho de agentes comunitários de saúde. Rev Cont Saúde. 2011; 11(20): 807-12.

21. Trindade LL, Gonzales RMB, Beck CLC, Lautert L. Cargas de trabalho entre os agentes comunitários de saúde. Rev Gaúcha Enfer. 2007; 28 (4): 473-79.

22. Oberg E, Frank E. Physicians' health practices strongly influence patient health practices. J R Coll Physicians Edinb. 2009; 39(4): 290.

23. McDowell N, McKenna J, Naylor P. Factors that influence practice nurses to promote physical activity. Br J Sports Med. 1997; 31(4): 308-13.

24. Frank E, Elon L, Hertzberg V. A quantitative assessment of a 4-year intervention that improved patient counseling through improving medical student health. Med Gen Med. 2007; 9(2): 58.

25. World Health Organization. Global recommendations on physical activity for health. Geneva: World Health Organization; 2010.

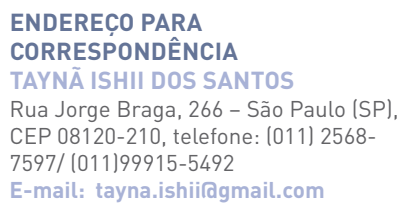

